\title{
Associação entre Má Oclusão e Condição de Saúde Bucal em Pacientes Atendidos nas Clínicas Odontológicas da Universidade do Estado do Rio Grande do Norte, Brasil
}

\author{
Association between malocclusion and oral health status in patients treated at the Dental Clinics
} of the State University of Rio Grande do Norte, Brazil

Asociación entre maloclusión y estado de salud oral en pacientes atendidos en las Clínicas Odontológicas de la Universidad Estatal de Rio Grande do Norte, Brasil

Rafael Ferreira de Paiva DIÓGENES

Departamento de Odontologia, Faculdade de Odontologia, Universidade do Estado do Rio Grande do Norte, 59300-00 Caicó - RN, Brasil https://orcid.org/0000-0002-6147-4627 Eloísa Cesário FERNANDES

Departamento de Odontologia, Faculdade de Odontologia, Universidade do Estado do Rio Grande do Norte, 59300-00 Caicó - RN, Brasil https://orcid.org/0000-0002-9330-1245

Francyle Simões Herrera SANCHES Departamento de Odontologia, Faculdade de Odontologia de Bauru, 17012901 São Paulo, Brasil https://orcid.org/0000-0002-9482-4371

Patrícia Bittencourt Dutra dos SANTOS Departamento de Orotodontia e Clínica Infantil, Faculdade de Odontologia, Universidade do Estado do Rio Grande do Norte, 59300-00 Caicó - RN, Brasil

https://orcid.org/0000-0003-3308-6376

\section{Resumo}

Introdução: Um alto índice de má oclusão e lesões de cárie é observado em crianças e adolescentes, porém a interação entre ambos ainda causa divergências no mundo científico. Objetivo: Investigar a possível associação entre má oclusão e condição de saúde bucal. Material e Métodos: O presente estudo transversal e retrospectivo foi realizado a partir da avaliação de prontuários de pacientes de 8 a 16 anos atendidos no setor das Clínicas Odontológicas da Universidade do Estado do Rio Grande do Norte - Brasil, entre os anos de 2013 a 2019. A amostra foi composta de 135 prontuários de pacientes divididos em 4 grupos distintos: pacientes com má oclusão de Classe I (Grupo 1), má oclusão de Classe II (Grupo 2), má oclusão de Classe III (Grupo 3) e pacientes com oclusão normal (Grupo 4). Para avaliar a condição de saúde bucal, foram coletadas informações sobre o CPO-D e seus componentes e para a identificação da má oclusão, utilizou-se a relação anteroposterior dos primeiros molares permanentes. Para verificar as possíveis associações entre o CPO-D e o tipo de má oclusão foi utilizado o teste Quiquadrado, e ainda o ANOVA one way para comparar a experiência de cárie entre os grupos, com significância de $5 \%$. Resultados: Não houve maior experiência de cárie dentro de cada grupo, assim como não houve diferença entre os grupos para a condição de saúde bucal. Conclusão: A condição de saúde bucal avaliada pela experiência de cárie não está associada à presença ou ausência de má oclusão quando a relação molar anteroposterior é avaliada.

Descritores: Má Oclusão; Saúde Bucal; Cárie Dentária; Criança.

\section{Abstract}

Introduction: A high rate of malocclusion and caries injuries is observed in children and adolescents, however the interaction between both still causes divergences in the scientific world. Objective: To investigate the possible association between malocclusion and oral health condition. Material and Methods: This cross-sectional and retrospective study was carried out based on the evaluation of medical records of patients aged 8 to 16 attended at the Dental Clinics of the State University of Rio Grande do Norte - Brazil, between the years 2013 to 2019 between the years 2013 to 2019. The sample consisted of 135 patient records divided into 4 distinct groups: patients with Class I malocclusion (Group 1), Class II malocclusion (Group 2), Class III malocclusion (Group 3) and patients with normal occlusion (Group 4). To assess the oral health condition, information was collected on the DMFT and its components and for the identification of malocclusion, the anteroposterior relationship of the first permanent molars was used. To check the possible associations between the DMFT and the type of malocclusion, the Chisquare test was used, as well as the one-way ANOVA to compare the caries experience between the groups, with a significance of $5 \%$. Results: There was no greater experience of caries within each group, just as there was no difference between groups for oral health condition. Conclusion: The oral health condition assessed by the caries experience is not associated with the presence or absence of malocclusion when the anteroposterior molar relationship is assessed.

Descriptors: Malocclusion; Oral Health; Dental Caries; Child.

\section{Resumen}

Introducción: Se observa una alta tasa de maloclusiones y lesiones por caries en niños y adolescentes, sin embargo la interacción entre ambas aún ocasiona divergencias en el mundo científico. Objetivo: investigar la posible asociación entre maloclusión y condición de salud bucal. Material y Métodos: Este estudio transversal y retrospectivo se realizó a partir de la evaluación de historias clínicas de pacientes de 8 a 16 años atendidos en las Clínicas Odontológicas de la Universidad Estatal de Rio Grande do Norte - Brasil, entre los años 2013 a 2019. La muestra estuvo conformada por 135 historias clínicas divididas en 4 grupos diferenciados: pacientes con maloclusión Clase I (Grupo 1), maloclusión Clase II (Grupo 2), maloclusión Clase III (Grupo 3) y pacientes con oclusión normal (Grupo 4). Para evaluar el estado de salud bucal se recogió información sobre el CPOD y sus componentes y para la identificación de maloclusión se utilizó la relación anteroposterior de los primeros molares permanentes. Para comprobar las posibles asociaciones entre el CPOD y el tipo de maloclusión se utilizó la prueba de Chicuadrado, así como el ANOVA de una vía para comparar la experiencia de caries entre los grupos, con una significancia del 5\%. Resultados: No hubo mayor experiencia de caries dentro de cada grupo, al igual que no hubo diferencias entre los grupos para la condición de salud bucal. Conclusión: El estado de salud bucal evaluado por la experiencia de caries no se asocia con la presencia o ausencia de maloclusión cuando se evalúa la relación molar anteroposterior.

Descriptores: Maloclusión; Salud Bucal; Caries Dental; Niño.

INTRODUÇÃO

A Organização Mundial de Saúde (OMS) recomenda que haja estudos constantes sobre os principais problemas de saúde bucal, já que seu perfil epidemiológico vem sofrendo contínuas transformações ao longo dos anos em todo 0 mundo ${ }^{1}$. Em uma escala de prioridades dos problemas bucais propostos pela própria OMS, a cárie dentária ocupa o primeiro posto, seguida pelas doenças periodontais e más oclusões ${ }^{1}$. 
A cárie dentária é uma doença bacteriana multifatorial que afeta e danifica os tecidos duros do dente, sendo uma das patologias bucais mais comuns na idade pediátrica $^{2}$. A ausência de tratamento pode levar invariavelmente a um aumento do tamanho da lesão em direção à polpa do dente, resultando em um aumento progressivo da inflamação pulpar acompanhada de sintomatologia dolorosa. Muitos são os fatores associados ao aparecimento das lesões cariosas, entretanto todos estão na dependência direta da presença de biofilme ${ }^{3}$.

A cárie é a principal causa de odontalgia e perda de dentes na população mundial, apesar de estar ocorrendo um declínio global nas últimas décadas ${ }^{3}$. A fluoretação das águas de abastecimento público, a adição de flúor nos dentifrícios, as alterações no padrão de consumo de açúcar, a melhora da higiene pessoal, incluindo a escovação, e o maior acesso à informação e à educação foram fatores essenciais para esse declínio ${ }^{4}$. Nas crianças, a prevenção tem um papel fundamental, pois permite evitar o aparecimento de lesões cariosas e dos seus efeitos, assim como instituir hábitos de higiene oral precocemente ${ }^{5}$.

As más oclusões apresentam uma elevada prevalência em ambas as dentições decídua e permanente ${ }^{6}$. O diagnóstico precoce de condições que influenciam 0 desenvolvimento normal da oclusão dentária é de extrema importância. Utilizando-se procedimentos de ortodontia preventiva e interceptativa, como manutenção de espaço ou a sua recuperação, o controle de hábitos bucais deletérios, e o tratamento da mordida cruzada e da mordida aberta, pode-se prevenir ou amenizar a severidade das oclusopatias. Quando essas más oclusões não são transitórias, ou seja, quando não sofrem autocorreção com a maturação da oclusão, é necessário um diagnóstico e intervenção precoces e adequados para que estas alterações não prejudiquem a evolução normal da dentição e do crescimento craniofacial ${ }^{7}$.

Os estudos que abordam a associação entre prevalência de lesões cariosas e severidade de más oclusões ainda são bastante divergentes no mundo científico. $O$ fato é que alguns estudos epidemiológicos sobre a prevalência de cárie dentária e de má oclusão têm sido apresentados na literatura; todavia, poucos são aqueles que se propuseram a avaliar simultaneamente a prevalência destes dois agravos em uma amostra adequada ${ }^{8}$. Além disso, não há relatos de estudos que utilizem as relações anteroposteriores para uma possível correlação com a condição de saúde bucal.

Dessa forma, esse estudo objetivou investigar a possível associação entre má oclusão e condição de saúde bucal, por meio da relação anteroposterior e índice CPOD, respectivamente. No intuito de tentar estabelecer um grupo de risco para o surgimento de lesões cariosas a depender do tipo de relação molar presente.

MATERIAL E MÉTODO

- Considerações éticas

Este projeto foi submetido à avaliação do Comitê de Ética em Pesquisa em Seres Humanos (CEP) da Universidade do Estado do Rio Grande do Norte (Protocolo n. 3.378.251).

- Amostra, critérios de elegibilidade, local do estudo

Tratou-se de um estudo transversal retrospectivo, no qual o cálculo do tamanho da amostra para a diferença de duas médias foi realizado considerando o poder do teste de $80 \%$ e o coeficiente de confiança de $95 \%$. Os valores referentes à estimativa do desvio padrão do CPOD-D foi de 1,29 e para a mínima diferença a ser detectada foi considerado 1,52 tomando por base estudo prévio ${ }^{9}$. O resultado mostrou que eram necessários 12 pacientes em cada grupo.

Foram avaliados todos os 1400 prontuários armazenados no arquivo da Universidade do Estado do Rio Grande do Norte. A amostra incluiu 135 pacientes sendo 58 do sexo feminino e 77 do sexo masculino com idades variando entre 8 e 16 anos e de baixa renda socioeconômica. Os critérios de exclusão adotados foram: prontuários sem a assinatura de autorização para exame, diagnóstico, documentação científica e/ou execução de tratamento e odontogramas sem assinatura do aluno e professor responsável. Os pacientes foram divididos em 4 grupos, sendo o grupo 1 composto por 16 pacientes com má oclusão de Classe I, grupo 2 composto por 45 pacientes com má oclusão de Classe II, grupo 3 composto por 21 pacientes com má oclusão de Classe III e o grupo 4 composto por 53 pacientes com oclusão normal.

\section{- Dados Coletados}

Informações oriundas dos prontuários foram coletadas por um único examinador.

1. Condição de saúde bucal: Foi utilizada a avaliação da experiência de cárie (CPO-D). Esse índice corresponde a soma das lesões de cárie, dentes perdidos por cárie e dentes restaurados. O índice CPO-D inclui um registro da presença / ausência de todos os dentes e é uma medida cumulativa da experiência de cárie. Foi feita a dicotomização da variável CPOD em 
dois níveis: leve (CPOD $\leq 2)$ e experiência de cárie severa $(C P O D \geq 3)$. $O$ ponto de corte de número 3 foi selecionado, porque as crianças com CPOD> 3 estavam acima do percentil 75 na nossa amostra de estudo, conforme estudo prévio $^{10}$. Todos os dados sobre o CPO-D foram coletados das fichas clínicas. 2. Má oclusão: Foram identificadas as relações anteroposteriores de molares (Classe I, II ou III), além de informações sobre trespasses verticais e horizontais. Pacientes com relação molar de classe I foram classificados no grupo de má oclusão de Classe I quando a relação molar estava acompanhada de mordida aberta ou cruzada. Enquanto, a relação molar de classe I na ausência dessas discrepâncias foi classificada no grupo de oclusão normal.

- Análise estatística

Realizou-se a estatística descritiva com o intuito de se obter as porcentagens das variáveis estudadas. O índice CPO-D e seus componentes foram comparados para cada grupo por meio do teste Qui-quadrado. A comparação intergrupos para todas as variáveis foi realizada por meio do teste de Análise de Variância a um critério (ANOVA one-way). A análise estatística foi realizada no programa Statistica, versão 10.0 e em todos os testes foram adotados níveis de significância de 5\%.

RESULTADOS

Previamente às análises comparativas entre os grupos, realizou-se a estatística descritiva com 0 intuito de se obter as porcentagens das variáveis estudadas (Tabela 1). Observou-se que a prevalência de pacientes com oclusão normal foi 39,6\%. Dentre as más oclusões, observou-se a maior prevalência de pacientes com má oclusão de Classe I de 11,85\%, Classe II 33,33\%, Classe II $15,5 \%$.

Tabela 1. Análise descritiva das variáveis investigadas

\begin{tabular}{|c|c|c|c|c|c|c|c|c|c|}
\hline & \multicolumn{2}{|c|}{ CPO-D (\%) } & \multirow[b]{2}{*}{$\mathbf{N}(\%)$} & \multicolumn{3}{|c|}{$\begin{array}{c}\text { Componentes } \\
\text { CPO-D (\%) }\end{array}$} & \multirow[b]{2}{*}{\begin{tabular}{|c|} 
Idade \\
(Média)
\end{tabular}} & \multirow[b]{2}{*}{$\begin{array}{c}\text { Sexo } \\
\text { Masculino }\end{array}$} & \multirow[b]{2}{*}{\begin{tabular}{|c|c|} 
Sexo \\
Femining
\end{tabular}} \\
\hline & $\begin{array}{c}\text { Leve } \\
(\%)\end{array}$ & $\begin{array}{c}\text { Severo } \\
(\%)\end{array}$ & & C (\%) & P(\%) & $\mathbf{R}(\%)$ & & & \\
\hline$\underline{\text { Classe I }}$ & 75 & 25 & 11,85 & 76,39 & 7,21 & 16,39 & 8,87 & 6 & 10 \\
\hline Classe II & 57,7 & 42,3 & 33,33 & 65,49 & 2,15 & 30,45 & 9,66 & 25 & 20 \\
\hline Classe III & 38,09 & 61,91 & 15,55 & 69,41 & 3,72 & 26,59 & 9,38 & 7 & 14 \\
\hline Oclusão Normal & 60,37 & 39,63 & 39,26 & 64,43 & 4,12 & 33,76 & 9,13 & 33 & 20 \\
\hline
\end{tabular}

Ao realizar o Teste Qui-Quadrado para avaliação da associação entre valores de CPO$\mathrm{D}$ e alterações anteroposteriores, constatou-se que não houve diferença significante entre o grau de CPO-D e a relação anteroposterior $(p=0,143)$ em todos os grupos (Tabela 2). Observou-se que para todos os grupos, com exceção do grupo 3, uma maior quantidade de pacientes apresentava uma experiência de cárie leve. Em geral, os pacientes do grupo 1 apresentaram um CPO-D menor que os demais grupos, embora essa diferença não tenha sido significante. Essa informação se repetiu para os componentes "Restaurados" e "Cariados". Em contrapartida, os maiores valores de dentes perdido foram encontrados no grupo 1, entretanto não houve diferença estatisticamente significante em relação aos outros grupos. Desse modo, comparação intergrupos para as variáveis do CPO-D de acordo com cada tipo de relação molar não mostrou diferença estatisticamente significante para a experiência de cárie quando os grupos foram comparados (Tabela 3).

Tabela 2. Teste Qui-Quadrado para avaliação da associação entre valores de CPO-D e alterações anteroposteriores.

\begin{tabular}{l|c|c|c}
\hline & \multicolumn{2}{|c|}{ CPO-D } & \multirow{2}{*}{ Total } \\
\hline Relação AP & LEVE (n) & SEVERO (n) & \\
\hline Classe I & 12 & 4 & 0,099 \\
\hline Classe II & 26 & 19 & 0,207 \\
\hline Classe III & 8 & 13 & 0,120 \\
\hline Oclusão normal & 32 & 21 & 0,146 \\
\hline X2 = 5,42; df= 2; $\mathbf{p = 0 , 1 4 3}$
\end{tabular}

Tabela 3. Comparação intergrupo para valores de CPO-D e seus componentes (ANOVA one-way)

\begin{tabular}{|c|c|c|c|c|c|}
\hline & $\begin{array}{l}\text { Classe I } \\
(\mathrm{n}=16)\end{array}$ & $\begin{array}{l}\text { Classe II } \\
(\mathrm{n}=45)\end{array}$ & $\begin{array}{l}\text { Classe III } \\
(\mathrm{n}=21)\end{array}$ & $\begin{array}{l}\text { Oclusão normal } \\
(\mathrm{n}=53)\end{array}$ & $\mathbf{P}$ \\
\hline & \multicolumn{5}{|c|}{ Média (DP) } \\
\hline Perdidos & $0,22(0,73)$ & $0,08(0,28)$ & $0,14(0,47)$ & $0,16(0,46)$ & 0,735 \\
\hline Restaurados & $0,50(0,92)$ & $1,13(1,49)$ & $1,00(1,48)$ & $1,31(1,38)$ & 0,185 \\
\hline Cariados & $2,33(3,58)$ & $2,43(2,19)$ & $2,61(2,43)$ & $2,50(2,60)$ & 0,986 \\
\hline CPO-D & $3,05(4,41)$ & $3,71(2,75)$ & $3,76(2,82)$ & $3,88(2,88)$ & $\frac{0,900}{0,794}$ \\
\hline
\end{tabular}

DISCUSSÃO

A possibilidade de uma associação entre má oclusão e cárie dentária pode ocorrer em ambas as direções, ou seja, a má oclusão como fator predisponente à cárie dentária e a cárie dentária como fator predisponente para a má oclusão. Esta possibilidade é uma característica do desenho transversal, que não permite a determinação de ordem de ocorrência dessas condições $^{11}$. Assim, além dos estudos que avaliam a má oclusão como variável independente e cárie dentária como resultado, há estudos demonstrando que os jovens com cárie dentária são mais propensos a apresentar má oclusão ${ }^{12-15}$.

Existem diversos estudos que utilizam o Índice de Estética Dentária (DAI) para avaliar as anormalidades dento-faciais, classificando as más oclusões e comparando-as com outras características, como a cárie. Este índice é composto por diversas características oclusais que são obtidas durante o exame clínico do paciente $^{16}$. O presente estudo, todavia, não utilizou o índice supracitado pelo fato de que os prontuários odontológicos utilizados como instrumento de coleta não contemplavam todas as informações exigidas para o preenchimento satisfatório. Por essa razão, optou-se por utilizar apenas a relação anteroposterior, pois trata-se de um componente bem relevante dentro do índice DAI ${ }^{16}$.

O conhecimento das mudanças 
anteroposteriores que ocorrem entre as dentaduras decídua, mista e permanente é muito importante para o clínico no planejamento do tratamento ortodôntico interceptativo precoce. O relacionamento anteroposterior entre os arcos dentários pode ser influenciado pelos hábitos bucais fuso prolongado da mamadeira, sucção de chupeta e dedo) e principalmente pela genética ${ }^{17,18}$. A literatura é vasta em considerar a relação anteroposterior em pesquisas sobre má oclusão ${ }^{19-24}$. Sendo assim, é possível considerar essa característica como bom preditor de más oclusões.

Já para a avaliação da experiência, o índice CPO de Klein e Palmer proposto em 1937 é universalmente aceito e preenche bem os requisitos necessários para um índice confiável e de fácil aplicação. A organização mundial da saúde utiliza esse índice como indicador básico de comparação para o estado de saúde bucal entre todas as populações do mundo, sendo adotado o valor de até 3 como satisfatório ${ }^{25}$.

$\mathrm{Na}$ avaliação descritiva dos dados coletados, observou-se uma maior presença do componente "Cariado" em todas os grupos desse estudo, corroborando com os resultados de Moura et al. ${ }^{26}$ que mostrou esse componente equivalendo a $66,3 \%$ do índice CPO-D. Entretanto, Porcelli et al. ${ }^{18}$ relataram que o item "restaurado" foi mais presente, ocupando $58,97 \%$ do total. Especula-se que essa discrepância seja devido à diferença em acesso ao serviço odontológico entre as comunidades estudadas. O item "perdidos", por outro lado, foi o menor no presente estudo e também em todos os outros encontrados na literatura (Tabela 1). Naturalmente, isso se deve ao fato de que a extração é a última alternativa frente aos problemas odontológicos.

A tabela 1 mostra a prevalência de má oclusão e oclusão normal para a população estudada. $\mathrm{Na}$ presente pesquisa, houve uma maior prevalência de pacientes com oclusão normal $(39,6 \%)$. Dentre as más oclusões, observou-se a maior prevalência de pacientes com má oclusão de Classe II $(33,33 \%)$, fato oposto ao que acontece com Porcelli et al. ${ }^{18} \mathrm{em}$ que é atribuída uma maior proporção à má oclusão classe I (75,8\%). Entretanto, a literatura mostra que dentre os portadores de má oclusão, embora a classe I seja a mais prevalente, pacientes com má oclusão de classe II são os que mais procuram o serviço odontológico devido ao envolvimento estético facial ${ }^{27}$.

A Tabela 2 mostra a distribuição de diferentes níveis de escores de CPO-D dentro de cada um dos grupos. Os resultados aqui presentes não mostraram diferença significante entre a experiência da cárie dicotomizada em leve ou severo para cada um dos grupos. Sendo assim, sugere-se que independente da relação anteroposterior, não há uma tendência de existir índices mais altos ou baixos de CPO-D.

O presente estudo encontrou uma média de CPO-D de 3,05, 3,71, 3,76, e 3,88 para os grupos relação molar classe, I, classe II, classe III e oclusão normal, respectivamente (Tabela 3). Esses valores estão acima do aceito pela $\mathrm{OMS}^{28}$, e ainda superiores aos encontrados nos estudos de Porcelli et al. ${ }^{17}$, no qual a média foi igual a 2,90 e por Moura et al. ${ }^{26}$, com resultado equivalente a 1,92. Obviamente, a presença de lesões de cáries e todas as repercussões em decorrência dela são influenciadas também por condições sócio-econômico-culturais, o que explica nossos resultados uma vez que os pacientes da presente amostra são de classe socioeconômica baixa.

A Tabela 3 mostra a comparação intergrupos para o CPO-D e todos seus componentes de acordo com cada tipo de relação molar. É possível perceber que não houve diferença estatisticamente significante para nenhum dos quesitos investigados, sugerindo que não há associação entre a experiência de cárie em todos os seus níveis e a má oclusão levando em consideração sua relação anteroposterior.

Entretanto, nosso estudo não corrobora com Singh et al. ${ }^{29}$ e Baskaradoss et al. ${ }^{30}$ os quais encontraram associação entre má oclusão e cárie dentária. Ainda não existe evidência científica para justificar a razão pela qual a má oclusão pode constituir um aumento de risco para a ocorrência de cárie, porém existem algumas hipóteses. A mais importante é que a má oclusão pode favorecer tanto o acúmulo de biofilme, como dificultar a sua remoção, e assim induzir o aparecimento de lesões de cárie. Essa maior retenção de biofilme está diretamente ligada à presença de apinhamento dentário.

Uma das justificativas plausíveis para a discrepância de nossos resultados com os observados na literatura se sustenta no fato de que o índice utilizado nos estudos é constituído não somente pela relação anteroposterior, mas também pelo apinhamento dentário. É pertinente ressaltar que este estudo não tinha como enfoque analisar o apinhamento, pelo fato de parte da faixa etária escolhida para análise se encontrar na dentadura mista. Nesse período, o apinhamento primário é considerado uma característica de normalidade. Dessa forma, esse apinhamento tem características transitórias, podendo se dissolver com o passar 
da idade diferentemente da relação anteroposterior, que é permanente. Entretanto, ressalta-se que tal fato não foi um viés em nossos resultados, pois a presença ou ausência de apinhamento estava distribuída aleatoriamente em todos os grupos de pacientes com má oclusão.

Ainda assim, este estudo obteve resultado semelhante ao encontrado em crianças iranianas, por Borzabadi-Farahani et al. ${ }^{11}$, de modo que esses autores também não encontraram associação entre as variáveis. Importante ressaltar que no referido estudo, além do índice DAI e CPO-D, também foi levado em consideração 0 fator socioeconômico.

A avaliação da condição socioeconômica é fundamental para estudos que abordam fatores de risco para cárie dentária. Pesquisas descobriram que indivíduos com menor nível socioeconômico têm maior prevalência de cárie dentária. Os trabalhos que tiveram resultados contrastantes com os nossos, ocultaram informações sobre essa condição. Ao interpretar essas descobertas, deve-se considerar que, devido a diferenças culturais, o nível socioeconômico variável e a metodologia que foi empregada não serem exatamente iguais aos usados em outros estudos, esses fatores podem explicar resultados diversos ${ }^{11}$. Seguindo essa linha de pensamento, e considerando que a renda familiar média é fragmentação em classe alta, classe média e classe baixa, o atual estudo tem mérito por utilizar pacientes advindos de uma realidade socioeconômica semelhante, uma vez que todos os pacientes são de baixa renda, o que reduz o risco de viés da pesquisa.

A utilização de um único banco de dados para a coleta representa uma limitação deste trabalho, bem como o uso de uma amostra por conveniência, pois não se pode extrapolar esses dados para toda a população da cidade. Além dessa limitação, este é um estudo transversal e, portanto, nenhuma relação de causalidade pode ser determinada. Muito embora, os trabalhos de natureza semelhante a este mostrados na literatura atual utilizaram uma amostra ligeiramente acima do que foi proposto nesta pesquisa ${ }^{17}$, a comparação deve ser feita com cautela, levando em consideração que 0 presente estudo avaliou a relação anteroposterior como preditor de má oclusão, não existindo, até o momento, trabalhos com parâmetros iguais para serem comparados diretamente.

Mesmo diante das limitações, o presente estudo mostrou ser bastante relevante em relação à temática abordada, visto que trouxe uma abordagem inédita de grande importância para a comunidade científica e para o clínico envolvido na formulação de políticas públicas e no planejamento de medidas preventivas contra esses dois problemas de saúde pública. Isso porque, a possibilidade de incluir mais um fator (relação molar) na identificação do grupo de risco de cárie ou ainda na tomada de decisão para intervalos de retorno dos pacientes é de grande valia, principalmente pensando em saúde pública. Reforça-se, entretanto, que novos estudos precisam ser feitos com uma metodologia mais aprimorada, amostras ampliadas e fatores identificadores de más oclusões mais precisos.

CONCLUSÃO

Não há associação entre má oclusão e condição de saúde bucal quando a relação molar anteroposterior e o CPO-D são utilizados como parâmetros de avaliação.

REFERÊNCIAS

1. Sousa RV, Pinto-Monteiro AKA, Martins CC, Granville-Garcia AF, Paiva SM. Malocclusion and socioeconomic indicators in primary dentition. Braz Oral Res. 2014; 28(1):1-7.

2. Bastos JL, Nomura LH, Peres MA. Dental pain, socioeconomic status, and dental caries in young male adults from southern Brazil. Cad. saúde publica. 2005;21:1416-23.

3. Castilho ARF, Mialhel FL, Barbosa TS, PuppinRontanil RM. Influência do ambiente familiar sobre a saúde bucal de crianças: uma revisão sistemática. J Pediatr. 2013;89:116-123.

4. Nowak AJ. Paradigm shift: infant oral health care-primary prevention. J Dent.2011;39:49-55.

5. Cavalcanti AL, Bezerra PKM, Alencar CRB, Moura C. Prevalência de Maloclusão em Escolares de 6 a 12 Anos de Idade em Campina Grande, PB, Brasil. Pesq Bras Odontoped Clín Integr. 2008;8(1):99-104.

6. Guzzo SC, Finkler M, Reibnitz Júnior $C$, Reibnitz MT. Ortodontia preventiva e interceptativa na rede de atenção básica do SUS: perspectiva dos cirurgiões-dentistas da Prefeitura Municipal de Florianópolis, Brasil. Ciênc saúde coletiva. 2014;19.

7. Dutra SR, Pretti H, Martins MT, Bendo CB, Vale MP. Impact of malocclusion on the quality of life of children aged 8 to 10 years. Dental Press $\mathrm{J}$ Orhod. 2018;23(2):46-53.

8. Borzabadi-Farahani A, Eslamipour F, Asgari I. Association between orthodontic treatment need and caries experience. Acta Odontol Scand. 2011;69:2-11.

9. Baskaradoss JK, Geevarghese A, Roger C, Thaliath A. Prevalence of malocclusion and its relationship with caries among school children aged 11 - 15 years in southern India. Korean J Orthod. 2013;43(1):35. 
10. Sá-Pinto AC, Rego TM, Marques LS, Martins CC, Ramos-Jorge ML, Ramos-Jorge J. Association between malocclusion and dental caries in adolescents: a systematic review and meta-analysis. Eur Arch Paediatr Dent. 2018; 19(2):73-82.

11. Gábris K, Márton S, Madléna M. Prevalence of malocclusions in Hungarian adolescents. Eur $\mathrm{J}$ Orthod. 2006; 28:467-70.

12. Frazão P, Narvai PC. Socio-environmental factors associated with dental occlusion in adolescents. Am J Orthod Dent Orthop. 2006; 16:129:809.

13. Nobile CGA, Paiva M, Fortunato L, Angelillo IF. Prevalence and factors related to malocclusion and orthodontic treatment need in children and adolescents in Italy. Eur J Public Health. 2007; 17:637-41.

14. Mtaya M, Brudvik P, Astrøm A. Prevalence of malocclusion and its relationship with sociodemographic factors, dental caries, and oral hygiene in 12- to 14-year-old Tanzanian schoolchildren. Eur J Orthod. 2009;31:467-76.

15. Kinnear TC, Taylor JR. Marketing research: an applied aproach. Mc Graw Hill. 1979.

16. Cons NC, Jenny J, Kohout FJ. Dai--the Dental Aesthetic Index. lowa City, lowa, USA: College of Dentistry, University of lowa, 1986. Print.

17. Mitchell, L. An Introdution to orthodontics. 3. ed. New York: Oxford University Press Inc; 2007.

18. Becky Chiang-Lin Liu, I-Chen Lee, Lun-Jou Lo, Ellen Wen-Ching Ko. Investigate the oral health impact and quality of life on patients with malocclusion of different treatment needs. Biomed J. 2019;42(6):422-29

19. Baume LJ. Uniform methods for the epidemiologic assessment of malocclusion. Am J Orthod. 1974; 63(3):251-72.

20. Farsi NMA.,Salama FS. Caracteristics of primary dentition occlusion in a group of Saudi children. Int J Paediatr Dent.1996; 6:253-259.

21. Infante PF. Malocclusion in the deciduous dentition in white, black and apache Indian children. Angle Orthod. 1975;3:213-18

22. Kerosuo $\mathrm{H}$. Occlusion in the primary and early mixed dentitions in a group of Tanzanian and Finnish children. J Dent Child. 1990;4:293-98.

23. Trottman A.; Elsbach HG. Comparison of malocclusion in preschool back and white children. Am J Orthod Dentofac Orthop. 1996; 110(1):69-82.

24. Woon KC. Primary dentition occlusion in Chinese, Indian and Malay groups in Malaysia. Aust Orthod.1988;10(3):183-85.

25. Klein H, Palmer CE. Dental caries in american indian children. Publ Helth Bull 1937;239:1-54.

26. Moura C, Cavalcanti AL. Malocclusion, dental caries and perceptions of aesthetics and function: an study of association. Rev odonto ciênc. 2007;22:256-62.
27. Martins MM, Araujo PS, Miguel JAM, Goldner MTA, Mendes AM. Tratamento orto-cirúrgico da classe II com avanço mandibular. Rev gaúch odontol. 2011;59(3):509-14.

28. Pigozzo MN, Laganá DC, Campos TN, Yamada MCM. A importância dos índices em pesquisa clínica odontológica: uma revisão da literatura. Rev Odontol Univ Cid São Paulo. 2008; 20(3):280-87.

29. Singh A, Purohit B, Sequeira P, Acharya S, Bhat M. Malocclusion and orthodontic treatment need measured by the dental aesthetic index and its association with dental caries in Indian schoolchildren. Community Dent Health. 2011;28(4):313-16.

30. Baskaradoss JK, Geevarghese A, Roger C, Thaliath A. Prevalence of malocclusion and its relationship with caries among school children aged 11-15 years in southern India. Korean $\mathrm{J}$ Orthod. 2013;43(1):35-41.

\section{CONFLITO DE INTERESSES}

Os autores declaram não haver conflitos de interesse

\section{AUTOR PARA CORRESPONDÊNCIA}

\section{Dr. Patrícia Bittencourt Dutra dos Santos}

Departamento de Orotodontia e Clínica Infantil Universidade do Estado do Rio Grande do Norte UERN

Av. Rio Branco, 725

59300-000 Caicó - RN, Brasil

Telefone comercial / fax: 5584999222420

e-mail: pati_bittencourt@hotmail.com 